\title{
OPTIMIZATION OF TREATMENT AND PREVENTION OF GENERALIZED PERIODONTAL DISEASES WITH THE USE OF TRANSGINGIVAL PHOTOACTIVED DISINFECTION
}

\author{
Valerii Burhonskyi \\ Department of Dentistry ${ }^{1}$ \\ burhonskyy@ukr.net \\ Sviatoslav Mykolaichuk \\ Department of Dentistry ${ }^{l}$
}

Valeriy Voitsekhovych

Department of Coherent and Quantum Optics

Institute of Physics of the National Academy of Sciences of Ukraine

46 Nauki ave., Kyiv, Ukraine, 03028

Moscow Institute of Physics and Technology (State University)

1 Klimentovsky ave., Moscow, Russian Federation, 115184

Vladimir Kholin ${ }^{2}$

Anna Reva ${ }^{2}$

${ }^{1}$ Shupyk National Medical Academy of Postgraduate Education

9 Dorohozhytska str., Kyiv, Ukraine, 04112

${ }^{2}$ Fotonika Plus Private Enterprise

50/12 Dakhnivska str, Cherkasy, Ukraine, 18009

\begin{abstract}
The aim of the study is to develop protocols for the treatment and prevention of generalized periodontal disease using a nozzle that designed for transgingival photosensitizer activation.

Methods. Analysis of available variants of irradiation nozzles for photoactivated disinfection. Circuit design and construction of authors' irradiation nozzle for transgingival photosensitizer activation.

Results. Based on the analysis of data, there was developed a nozzle design that allows treating periodontium areas within 4-6 teeth, evenly distributes the required power of laser radiation, and can be used in hard-to-reach areas of the oral cavity. Based on the above calculations, a nozzle for transgingival photosensitizer activation was created (jointly with Fotonika Plus, PE). To optimize the PAD procedure, there was created a protocol of transgingival photoactivated disinfection to be applied at the stage of professional oral hygiene, using the created irradiation nozzle NOU-9 of authors' design, and diode $2 \mathrm{~W}$ laser LIKA-surgeon with a wavelength of $660 \mathrm{~nm}$. The result is the accelerated procedure of transgingival photoactivated disinfection.

Conclusions. The development of photoactivated disinfection technology allows supplementing the traditional treatment of generalized periodontal diseases. Studies of non-invasive, transgingival method of photosensitizer activation have recently become popular. This, in turn, carries a lower probability of cross-infection and less traumatization of periodontal tissues during the manipulation. The use of PAD cannot lead to resistance of the microflora in contrast to pharmacological antibacterial preparations. Carrying out PAD does not require any complex manual skills, the purchase of expensive equipment, provides an opportunity for widespread introduction of technology. The use of the therapeutic complex and protocols of PAD that were developed can significantly reduce the duration of the procedure, resulting in improved comfort for both the doctor and the patient. The NOU-9 irradiation nozzle allows reaching hard-to-reach areas of the oral cavity, distributing laser radiation efficiently and evenly on periodontal tissues. The LIKA-surgeon $2 \mathrm{~W}$ laser with a wavelength of $660 \mathrm{~nm}$ provides the operating parameters required to activate the photosensitizer. Due to low-intensity radiation, it is possible to enhance regenerative processes in the periodontium after traumatic intervention. Clinical studies on the antibacterial efficacy of the technology are ongoing.
\end{abstract}

Keywords: periodontitis, transgingival photoactivated disinfection, irradiation nozzle, therapeutic complex, protocol. 


\section{Introduction}

According to statistics, generalized periodontal diseases are the most common dental pathology after dental caries, affecting one third of the world's population [1].

Important elements in the fight against this pathology are the mechanical removal of dental plaque and subsequent drug therapy, which is mainly aimed at combating periodontal pathogenic microflora [2]. However, the acquired resistance of microorganisms to both antibiotics and antiseptic drugs used in dental practice has increasingly being recorded [3].

In addition, during scaling and curettage, microorganisms in the gingival sulcus or periodontal pocket enter the bloodstream, causing bacteremia that cannot be treated with antiseptic drugs [4].

Considering alternative to pharmacotherapy methods of exposure to periodontal pathogens, the technology of photoactivated disinfection produces good results, which is based on the interaction of laser radiation and a special substance - photosensitizer [5]. A number of studies indicate the effectiveness of this technology as an adjunct to traditional periodontal treatment [6]. In addition to antibacterial effects, laser radiation used to activate the photosensitizer also has significant wound healing and biostimulating effects [7].

To bring the energy of laser radiation from the laser to the periodontal tissues with previously introduced photosensitizer, they use quartz or polymer optical fibres. Their terminal part is either treated in a certain way, or is a specially designed nozzle with optical elements [8]. The literature provides evidence that some types of fibres for photoactivation can injure periodontal tissues, impairing the therapeutic effect. Their use is also time-consuming, which makes it impossible to widely use the technology [9].

Recent studies indicate that laser radiation with a wavelength of $670 \mathrm{~nm}$ can activate the photosensitizer not only by direct delivery to the affected area, but also in transgingival way [10]. Thus, using the nozzle that developed for transgingival irradiation, can achieve activation of the photosensitizer not only in the periodontal pocket, but also in the depth of the gums.

The aim of the study is to study the possibility of optimizing the treatment and prevention of generalized periodontal diseases by performing transgingival photoactivated disinfection in patients with both healthy periodontium and generalized periodontal pathology during scheduled professional oral hygiene, using the designed therapeutic complex.

\section{Materials and methods}

There is a wide variety of irradiation nozzles, which can be arranged into several main groups by types:

- Nozzles which are a specially treated part of a fibre (quartz, polymer), or a group of fibres [11].

- Nozzles which are scattering optical elements [12].

- Nozzles that are a glass fibre [13] (Fig. 1).

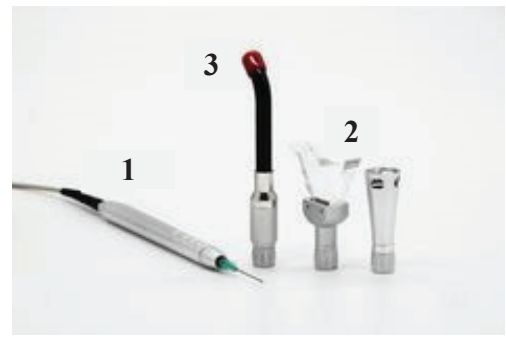

Fig. 1. The main types of irradiation nozzles

Having these types of nozzles available, and widely practicing the technology of photoactivated disinfection for the treatment of generalized periodontal diseases, there were identified certain shortcomings on these designs:

1. Nozzles of the first type are convenient for penetration into periodontal pockets, but treatment of all dentition in cases of the generalized periodontal diseases is rather labour-consuming for 
the doctor, takes a lot of time, painful sensations are possible in the course of the procedure because of pricks by a sharp tip of optical fibre.

2. Nozzles of the second type allow reducing time of procedure of photoactivated disinfection, while allowing to treat only a vestibular part of a periodontium, power of laser radiation is not evenly distributed (that is important for photosensitizer activation), they do not provide for full penetration.

3. Nozzles of the third type allow reaching different groups of teeth and irradiating periodontal tissues from the oral side too, but their range is limited to 1-2 teeth that is also time-consuming.

Based on the analysis of the data, there was developed a nozzle design that allows treating periodontal areas within 4-6 teeth, evenly distributes the required power of laser radiation, and allows working in hard-to-reach areas of the oral cavity. The following required parameters were also calculated:

- the approximate appearance and size of the light spot on the gums is shown in the figure below;

- distance from the emitter to the irradiation zone $\leq 1 \mathrm{~cm}$;

- power density on the gums $-125 \mathrm{~mW} / \mathrm{cm}^{2}$ (Fig. 2).

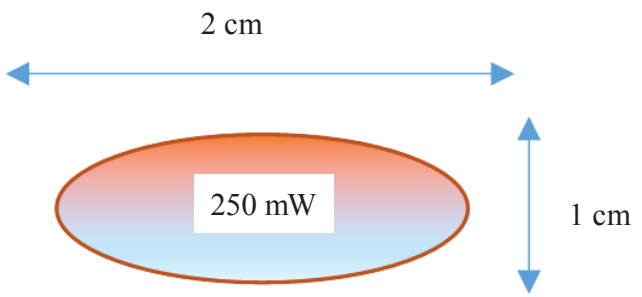

Fig. 2. Radiation spot on the gums

There are 2 options for the use of this tool:

1. A cylindrical scattering lens shall be installed at the tool point, which additionally expands the radiation from the fibre up to $2 \mathrm{~cm}$ on the gums in one projection, and without additional expansion in the perpendicular direction (only due to the angle of emergence from the fibre).

2. The tool shall be equipped by a bundle - not one fibre, but 5 , for example, where laser radiation is introduced. At the output of the tool, the fibres are distributed in one plane with a slight difference in angle.

Based on the above calculations, a nozzle for transgingival photosensitizer activation was designed and created (jointly with Photonika Plus, PE) $($ Fig. 3, 4). To achieve the required power density $\left(125 \mathrm{~mW} / \mathrm{cm}^{2}\right)$ of laser radiation on the gums, the $2 \mathrm{~W}$ LIKA-surgeon diode laser with a wavelength of $660 \mathrm{~nm}$ was use. To achieve the required power density $\left(125 \mathrm{~mW} / \mathrm{cm}^{2}\right)$ of laser radiation on the gums, we used the $2 \mathrm{~W}$ LIKA-surgeon diode laser with a wavelength of $660 \mathrm{~nm}$ [12].

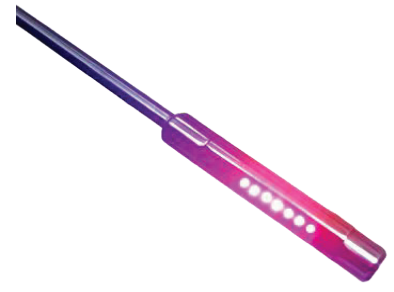

Fig. 3. Irradiation head NOU-9 nozzle

Fig. 4. The designed NOU-9 nozzle 


\section{Results}

To optimize the PAD procedure, a protocol for transgingival photoactivated disinfection at the stage of professional oral hygiene was created:

a) before the procedure of professional hygiene according to the protocol, a solution of photosensitizer (methylene blue, Fotolon) is introduced into the gingival sulcus or periodontal pocket with a syringe and endodontic needle in the area of all available teeth, until the sulcus or pocket is completely filled;

b) upon introducing photosensitizer, its exposure is held depending on the degree of damage to periodontal tissues:

- for 2 minutes - healthy periodontium, catarrhal gingivitis, ulcerative gingivitis;

- for 5 minutes - generalized periodontitis of degree I and II;

- for 7 minutes - generalized periodontitis of degree III;

c) this is followed by professional oral hygiene performed according to the protocol.

The oral cavity is conventionally divided into 6 sectors (Fig. 5):

1. Upon completion of professional hygiene, sector-by-sector irradiation of the oral cavity (from the oral and vestibular sides) is performed with the NOU-9 nozzle connected to a laser with a wavelength of $660 \mathrm{~nm}$ at a power of $1.5 \mathrm{~W}$ in pulsed mode (Fig. 6).

2. Duration of irradiation for different types of pathology:

- 1 minute (30 seconds oral side, 30 seconds vestibular one) per sector - healthy periodontium, catarrhal gingivitis, ulcerative gingivitis;

-2 minutes per sector ( 1 minute oral side, 1 minute vestibular one) -generalized periodontitis of degree I and II;

- 4 minutes per sector ( 2 minutes oral side, 2 minutes vestibular one) - generalized periodontitis of degree III.

The procedure is followed by recommendations for further general treatment and, if necessary, for repeated PAD procedures.

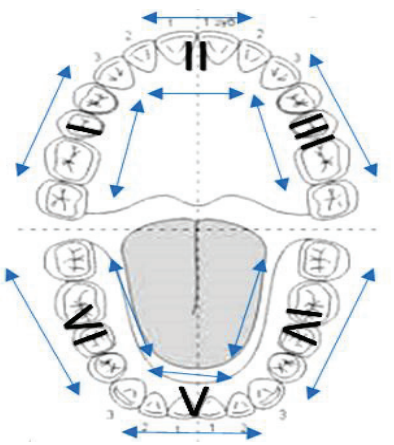

Fig. 5. Division into 6 sectors

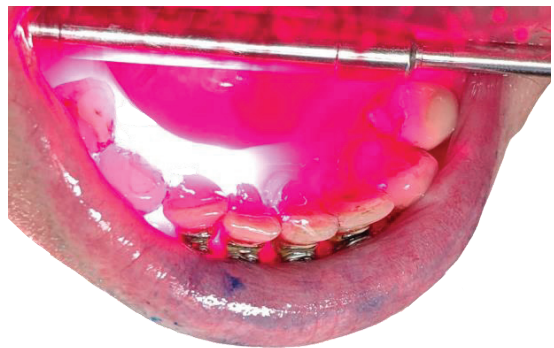

Fig. 6. Irradiation of the frontal sector

\section{Discussion}

In recent years, PAD has increasingly being introduced into dental practice in the treatment of periodontal pathology. However, the potential of this technology is not fully revealed. According to the group of studies, PAD has a more effective impact on some periodontal pathogens than 
scaling and curettage. Besides, this type of disinfection can inactivate a certain group of cytokines in the host organism, which can provide a more favourable environment for healing. Moreover, there are clinical benefits of using PAD in periodontology.

In vitro studies showed a destructive effect on periodontal pathogenic microflora both in suspension and in the composition of dental plaque, which indicates the reasonability of PAD after scaling to prevent reinfection, because the ultrasound destruction of dental plaque in the mouth result in forming a suspension of microorganisms released from its composition. The available positive results of transgingival photoactivated disinfection give impetus to apply this technology more widely.

According to some data, laser radiation used to activate the photosensitizer has significant wound healing and biostimulating effects [12], which is important in rehabilitation after interventions. However, there are data on the insignificant or unproven effectiveness of photoactivated disinfection [13]. However, this technology has an advantage over the use of traditional antibacterial drugs, namely the inability of the microflora to become resistant to PAD, as has recently become common in the form of pharmacological resistance.

Study limitations. The study did not include individuals with transitional dentition, edentulous jaws, malignant processes in the maxillofacial area, infectious diseases in the active stage, periodontal diseases of non-communicable etiology. Patients with individual sensitivity to photosensitizer components were not involved in the study.

Prospects for further research. It is planned to study the antibacterial effectiveness of the proposed technology of photoactivated disinfection by taking the material three times: immediately after the procedure, and 7 and 14 days after it. Sampling will be performed with a paper point from gingival crevice (apparently healthy) or periodontal pocket (periodontitis) in the area of teeth $1.6,4.1,3.6$ to study the quantitative presence of DNA of periodontal pathogens (PCR). The conclusions will be drawn based on the results of the study.

\section{Conclusions}

Studies of non-invasive, transgingival method of photosensitizer activation have recently become popular due to the development of microflora resistance to antibacterial drugs. An important factor is the convenience of laser radiation delivery with the required radiation density and power to the target tissue. The existing types of irradiating nozzles have a number of disadvantages, including long duration of the procedure, non-ergonomics, and pain during manipulation.

The developed protocol of PAD with the use of NOU-9 irradiating nozzle and the LIKA-surgeon $2 \mathrm{~W}$ laser (wavelength of $660 \mathrm{~nm}$ ) optimizes the manipulation. The use of this technology can significantly reduce the duration of the procedure and, as the result, make it more convenient to the doctor and the patient. The NOU-9 irradiation nozzle can involve hard-to-reach areas of the oral cavity, distribute laser radiation efficiently and evenly on periodontal tissues. The LIKA-surgeon $2 \mathrm{~W}$ laser provides the power required to activate the photosensitizer. Low-intensity radiation makes it possible to enhance regenerative processes in the periodontium after traumatic intervention. Clinical studies on the antibacterial efficacy of the technology are ongoing.

\section{Conflict of interests}

The authors declare that they have no conflict of interests

\section{References}

[1] Nazir, M. A. (2017). Prevalence of periodontal disease, its association with systemic diseases and prevention. International Journal of Health Sciences, 11 (2), 72-80.

[2] Mazur, I. P., Peredrii, V. A., Dulko, S. V. (2010). Pharmacological chemicals for the local treatment of periodontal tissues. Parodontolohiia, 5, 47-52.

[3] Kampf, G. (2016). Acquired resistance to chlorhexidine - is it time to establish an «antiseptic stewardship» initiative? Journal of Hospital Infection, 94 (3), 213-227. doi: http://doi.org/10.1016/j.jhin.2016.08.018

[4] Rams, T. E., Degener, J. E., van Winkelhoff, A. J. (2014). Antibiotic Resistance in Human Chronic Periodontitis Microbiota. Journal of Periodontology, 85 (1), 160-169. doi: http://doi.org/10.1902/jop.2013.130142 
[5] Coovadia, Y., Vayej, A. C., Maharaj, B. (2012). An investigation of the frequency of bacteraemia following dental extraction, tooth brushing and chewing: cardiovascular topics. Cardiovascular Journal of Africa, 23 (6), 340-344. doi: http:// doi.org/10.5830/cvja-2012-016

[6] Parker, S. (2013). The use of diffuse laser photonic energy and indocyanine green photosensitiser as an adjunct to periodontal therapy. British Dental Journal, 215 (4), 167-171. doi: http://doi.org/10.1038/sj.bdj.2013.790

[7] Novaes, A. B., Schwartz-Filho, H. O., de Oliveira, R. R., Feres, M., Sato, S., Figueiredo, L. C. (2011). Antimicrobial photodynamic therapy in the non-surgical treatment of aggressive periodontitis: microbiological profile. Lasers in Medical Science, 27 (2), 389-395. doi: http://doi.org/10.1007/s10103-011-0901-6

[8] Obradović, R., Kesić, L., Mihailović, D., Antić, S., Jovanović, G., Petrović, A., Peševska, S. (2012). A histological evaluation of a low-level laser therapy as an adjunct to periodontal therapy in patients with diabetes mellitus. Lasers in Medical Science, 28 (1), 19-24. doi: http://doi.org/10.1007/s10103-012-1058-7

[9] Passanezi, E., Damante, C. A., de Rezende, M. L. R., Greghi, S. L. A. (2014). Lasers in periodontal therapy. Periodontology 2000, 67 (1), 268-291. doi: http://doi.org/10.1111/prd.12067

[10] Husejnagic, S., Lettner, S., Laky, M., Georgopoulos, A., Moritz, A., Rausch-Fan, X. (2019). Photoactivated disinfection in periodontal treatment: A randomized controlled clinical split-mouth trial. Journal of Periodontology, 90 (11), $1260-1269$. doi: http://doi.org/10.1002/jper.18-0576

[11] Wenzler, J. S., Böcher, S., Frankenberger, R., Braun, A. (2019). Feasibility of transgingival laser irradiation for antimicrobial photodynamic therapy. Photodiagnosis and Photodynamic Therapy, 28, 75-79. doi: http://doi.org/10.1016/j.pdpdt.2019.08.030

[12] Ronay, V., Buchalla, W., Sahrmann, P., Attin, T., Schmidlin, P. R. (2013). In vitroevaluation of the oxidation efficacy of transgingival photodynamic therapy. Acta Odontologica Scandinavica, 71 (5), 1216-1220. doi: http://doi.org/10.3109/00016357.2012.757639

[13] Chatzopoulos, G. S., Doufexi, A. E. (2016). Photodynamic therapy in the treatment of aggressive periodontitis: A systematic review. Medicina oral, patologia oral y cirugia bucal, 21 (2), e192-e200. doi: http://doi.org/10.4317/medoral.21046

Received date 16.11.2020

(C) The Author(s) 2021

Accepted date 26.01.2021

Published date 09.02.2021

This is an open access article under the CC BY license (http://creativecommons.org/licenses/by/4.0).

How to cite: Burhonskyi, V., Mykolaichuk, S., Voitsekhovych, V., Kholin, V., Reva, A. (2021). Optimization of treatment and prevention of generalized periodontal diseases with the use of transgingival photoactived disinfection. EUREKA: Health Sciences, 2 , 31-36. doi: http://doi.org/10.21303/2504-5679.2021.001614 